\title{
Enfermedad de Behçet en Chile: Análisis clínico de 44 casos
}

\author{
Pamela W urmann ${ }^{1}$, G onzalo Díaz ${ }^{2}$, Francisca Sabugo ${ }^{1}$, \\ Lilian Soto ${ }^{1}$, Federica Solanes ${ }^{a}$, Sandra Pino ${ }^{1}$, G uillermo \\ Merino $^{3}$, Juan Ignacio Verdaguer ${ }^{4}$, Francisco Villarroel $^{4}$, \\ Miguel Cuchacovich ${ }^{1}$.
}

\section{Retrospective review of 44 Chilean patients with Behçet disease}

Background: Behçet's disease (BD) is a rare multisystemic inflammatory disease that is potentially disabling and may cause death. Aim: To describe the characteristics of BD patients from two Chilean centers. Patients and method: Retrospective review of the clinical records of patients with $\mathrm{BD}$ attended in two rheumatology services between 1985 and 2007. The "Behçet's Disease Research Committee of Japan" (BDCJ) and the "International Study Group for Behçet's Disease" (ISG) diagnostic criteria were applied. Results: We found 44 cases ( 25 males), diagnosed as BD. The mean age at the onset of symptoms was $26 \pm 12$ years. According to BDCJ criteria, 13 patients had complete BD, 24 had incomplete BD and 7 had a suspected BD. Thirty two patients fulfilled the ISG criteria. Forty two patients (95\%) had oral ulcers, $33(75 \%)$ had genital ulcers and 29 (66\%) had ophthalmological involvement. Eleven and three patients had symptoms of central and peripheral nervous system involvement, respectively. No gender differences were detected. Conclusions: The clinical characteristics of these patients were similar to those described abroad, except for a higher frequency of peripheral nervous system involvement and a lower rate of arthritis (Rev Méd Chile 2009; 137: 1333-40).

(Key w ords: Behçet syndrome; Oral ulcer; Uveitis)

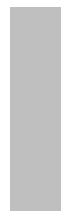

\footnotetext{
Recibido el 14 de octubre, 2008. Aceptado el 7 de septiembre, 2009.

${ }^{1}$ Sección de Reumatología, Departamento de Medicina, Hospital Clínico de la Universidad de Chile. Santiago de Chile. ${ }^{2}$ Becado de Medicina Interna, Hospital Clínico, Campus Norte, Universidad de Chile. ${ }^{3}$ Centro Oftalmológico Luis Pasteur. ${ }^{4}$ Fundación Oftalmológica Los Andes.

${ }^{a}$ Interna de Medicina
}

L a enfermedad de Behçet (EB) es una enfermedad inflamatoria crónica, recurrente, multisistémica, de etiología desconocida que se caracteriza por períodos de remisión y de exacerbación de frecuencia y duración impredecibles. La primera

$\overline{\text { Correspondencia a: Dr. Miguel Cuchacovich T. San Pío X }}$ 2460 Of. 607. Providencia. Fono: 56-2-2048821. Fax: 56-22312395. E mail: mcuchacovich@redclinicauchile.cl descripción de su sintomatología, realizada por Hipócrates, se remonta al siglo V a.C. Posteriormente, el oftalmólogo griego Benedictos Adamantiades en $1931 \mathrm{y}$, en forma independiente, el dermatólogo turco Hulusi Behçet en 1937 completaron su descripción ${ }^{1}$. Es más frecuente entre el este asiático y el Mediterráneo, por lo que también se la conoce como enfermedad de la ruta de la seda. 
Su incidencia es muy variable, desde 1 en 1.000 en Turquía, 1 en 10.000 en Japón, Corea, Irán y Arabia Saudita, hasta 1 en 500.000 en el Reino Unido, e incluso 1 en 1 millón en Estados Unidos de Norteamérica ${ }^{2}$. Afecta predominantemente a adultos jóvenes y tradicionalmente se describía como más frecuente en hombres que en mujeres, sin embargo en los últimos años esta diferencia ha disminuido acercándose a una relación $1: 1^{2}$

Los órganos que se ven más afectados son la piel, membranas mucosas, ojos, articulaciones, sistema vascular, pulmones, tracto gastrointestinal y sistema nervioso. La etiología y patogenia de la EB son desconocidas, pero el inicio de la enfermedad parece ser gatillado por factores ambientales en pacientes portadores de una susceptibilidad genética particular. El antígeno mayor de histocompatibilidad HLA-B51 es el marcador genético que más se asocia con la EB en los distintos grupos étnicos, con un riesgo relativo que oscila entre 1,38 y $20,70^{3}$. Dentro de los factores ambientales involucrados se han postulado los agentes infecciosos. Ha sido publicado que el Estreptococo sanguis se encuentra frecuentemente en la flora oral de pacientes con EB. El ácido lipoteicoico (LTA) es un antígeno estreptocócico capaz de inducir activación de linfocitos y uveítis anterior en modelos experimentales. Hemos demostrado recientemente que los pacientes portadores de EB activa presentan niveles elevados de IgG anti-LTA capaces de activar complemento in vitro. Del mismo modo, el LTA estimula la secreción de interleuquina 8 en células mononucleares de pacientes con EB activa ${ }^{4}$. El fenómeno patológico subyacente al compromiso visceral es una vasculitis. La EB puede afectar arterias y venas de todos los tamaños, pero el compromiso venoso es el más frecuente. Aproximadamente 25\% de los pacientes presentan trombosis venosa ${ }^{5}$.

La EB no tiene manifestaciones clínicas patognomónicas, ni exámenes de laboratorio específicos, por lo que el diagnóstico se realiza en base a criterios clínicos predefinidos según distintos grupos de estudio. Dentro de éstos, los más utilizados son los de Behçet's Disease Research Committee of Japan $(B D C J)^{6}$, y los del International Study Group for Behçet's Disease (ISG) ${ }^{7}$. (Anexos a disposición de los lectores que los soliciten al autor corresponsal).
Es generalmente aceptado que en Chile, la EB es de muy baja frecuencia, pero no se dispone de datos epidemiológicos precisos. Tampoco existen descripciones de los síntomas más frecuentes ni de la forma de presentación en cohortes locales.

Por lo anterior se planteó como objetivos de este estudio, describir las características clínicas de la EB en un grupo de pacientes chilenos que cumplían con los criterios diagnósticos del BDCJ en 2 centros de derivación terciaria de alta complejidad. Se aplicaron además los criterios diagnósticos del ISG con el fin de definir qué instrumento presentaba un mejor rendimiento en nuestra población. Se buscó diferencias clínicas por género y se realizó una comparación con otras cohortes internacionales de EB.

\section{PACIENTES Y MÉTODOS}

Se trata de un estudio retrospectivo, realizado en dos centros de derivación terciaria reumatológica. Se analizaron las fichas de pacientes con diagnóstico clínico de EB según las bases de datos locales, entre los años 1985 y 2007. A todos los pacientes se les aplicaron los criterios de BDCJ y los ISG con datos obtenidos de la ficha. Se encontraron 50 registros, de los cuales 6 fueron descartados por no cumplir con ninguno de los 2 criterios, por lo que finalmente se seleccionaron 44 pacientes de los que se describen las manifestaciones clínicas registradas durante su evolución. Se realizó una comparación de nuestra serie de acuerdo a los criterios ISG y BDCJ y posteriormente con cohortes de España y Grecia.

El análisis estadístico se realizó con una prueba t de Student para proporciones.

\section{Resultados}

En la búsqueda se obtuvieron 44 pacientes que cumplieron con los criterios diagnósticos de EB y se revisaron las fichas clínicas registrando las manifestaciones clínicas durante la evolución.

La edad promedio de comienzo de los síntomas fue de 26,29 $\pm 11,76$ años, con rangos que variaron entre 11 y 56 años; 19 eran mujeres $(43,2 \%)$ y 25 eran hombres $(56,8 \%)$ ( $\mathrm{p}=0,28)$. El tiempo de seguimiento promedio fue de 8,7 años con rangos que variaron entre 2,2 y 20. 
Entre los síntomas típicos, las úlceras orales fueron las que se presentaron con mayor frecuencia, seguidas por las úlceras genitales, el compromiso cutáneo y en menor porcentaje la afección oftalmológica (Tabla 1).

Las manifestaciones oftalmológicas, las características de las manifestaciones cutáneas y la frecuencia de los síntomas adicionales durante la evolución de la enfermedad se presentan en las Tablas 2, 3 y 4, respectivamente.

Dentro del compromiso ocular, la forma más frecuente de presentación en nuestro estudio, así como en series internacionales, fue la afección del segmento posterior ya sea como uveítis posterior o pan uveítis, con o sin vasculitis retinal ${ }^{8}$. Su curso fue severo y crónico requiriendo el uso de esteroides e inmunosupresores en todos los sujetos para el adecuado control de la enfermedad. La ciclosporina A fue muy eficaz en el control del compromiso ocular en la mayoría de los pacientes
(20/28), 3 pacientes requirieron del uso de ciclosporina A y azatioprina en combinación y 5 requirieron del uso de infliximab con buena respuesta en todos 9 . Por otra parte, la presencia de edema macular cistoide ( 2 pacientes) constituyó un signo de mal pronóstico, que no revirtió a pesar del tratamiento.

De aquellos que presentaron artritis 8 fueron oligoartritis y 2 poliartritis. En todos los casos esta manifestación fue de curso crónico y de difícil tratamiento, incluso un paciente requirió infliximab para su control, con buen resultado; sin embargo, al intentar discontinuar esta droga el paciente recayó a las 8 semanas postsuspensión.

El compromiso del sistema nervioso central (SNC) se manifestó como neurovasculo-Behçet en 7 pacientes, correspondiendo 4 a accidentes vasculares encefálicos y 3 a trombosis de senos venosos cerebrales. Cuatro pacientes tuvieron manifestaciones tipo neuro-Behçet propiamente tal ( $\sin$ com-

Tabla 1. Frecuencia de síntomas típicos en el grupo estudiado durante la evolución de la enfermedad de Behçet

\begin{tabular}{|lcc|}
\hline Síntoma & $\mathbf{n}$ & $\%$ \\
\hline Úlceras orales recurrentes & 42 & 96 \\
Úlceras genitales & 33 & 75 \\
Lesiones cutáneas & 29 & 66 \\
Compromiso oftalmológico & 28 & 64 \\
\hline
\end{tabular}

n =número de pacientes.

Tabla 2. C aracterísticas del compromiso oftalmológico en el grupo estudiado durante la evolución de la enfermedad de Behçet $(\mathbf{n}=\mathbf{2 8})$

\begin{tabular}{|lrr|}
\hline Tipo & $\mathbf{n}$ & $\%$ \\
\hline Uveítis anterior exclusiva & 7 & 25 \\
Uveítis posterior exclusiva & 7 & 25 \\
Panuvétis & 13 & 46 \\
Vasculitis retinal & 8 & 29 \\
Hipopion & 1 & 4 \\
Edema macular & 2 & 7 \\
\hline
\end{tabular}

$\mathrm{n}=$ número de pacientes. 


\section{Tabla 3. Frecuencia de lesiones cutáneas en el grupo estudiado durante la evolución de la enfermedad de Behçet} ( $\mathrm{n}=29$ )

\begin{tabular}{|lrl|}
\hline Tipo & $\mathbf{n}$ & $\%$ \\
\hline Eritema nodoso & 15 & 52 \\
Tromboflebitis superficial & 7 & 24 \\
Lesiones papulopustulares & 17 & 59 \\
\hline
\end{tabular}

$\mathrm{n}=$ número de pacientes.

Tabla 4. Síntomas adicionales en el grupo estudiado durante la evolución de la enfermedad de Behçet ( $\mathrm{n=44)}$

\begin{tabular}{|lrr|}
\hline Síntoma & $\mathbf{n}$ & $\%$ \\
\hline Artritis & 10 & 23 \\
Epididimitis & 4 & 9 \\
Úlceras 1́leo-cecales & 0 & 0 \\
Sistema vascular & 13 & 30 \\
SNC & 11 & 25 \\
Neurovasculo-Behçet & 7 & 15,9 \\
Neuro-Behçet & 4 & 9,1 \\
SNP & 3 & 7 \\
\hline
\end{tabular}

n =número de pacientes, SNC =sistema nervioso central, SNP = sistema nervioso periférico.

promiso macrovascular): meningitis aséptica en 2 casos, 1 de enfermedad desmielinizante y 1 de leucoencefalopatía posterior reversible.

Cabe destacar que 3 pacientes presentaron alteraciones de sistema nervioso periférico (SNP), caracterizadas por polineuropatía sensitivo motora distal de 4 extremidades en 2 de ellos y mononeuritis múltiple en uno.

En cuanto al compromiso vascular, 3 pacientes presentaron trombosis venosa profunda (TVP), 3 vasculitis sistémica y 7 tromboflebitis superficial. Un paciente presentó una hemorragia alveolar durante el curso de su vasculitis, falleciendo a pesar del tratamiento inmunosupresor. En este grupo, no se incluyeron los pacientes con trombosis arteriales o venosas de SNC, porque ya fueron mencionados dentro del compromiso del SNC.

No hubo diferencias estadísticamente significativas al comparar las características clínicas entre hombres y mujeres (Tabla 5).
De acuerdo a los criterios BDCJ, de los 44 casos, 13 cumplían criterios completos de enfermedad, 24 incompletos y 7 estuvieron en rango de sospecha. Al aplicar los criterios del ISG sólo 32 de los 44 cumplían con los criterios de enfermedad.

Cinco de los pacientes con criterios BDCJ ya fueran completos o incompletos, no cumplieron los criterios ISG. En uno de ellos la causa de la exclusión fue la ausencia de úlceras orales y en 4 la presencia de sólo 2 criterios. Al analizarlos desde el punto de vista de los criterios BDCJ, se observa que estos pacientes cumplían criterios de EB incompleto debido a la presencia de lesión ocular típica más otro síntoma típico en 4 casos y 2 síntomas típicos más 2 síntomas adicionales en 1 caso. Es destacable que ninguno de estos pacientes presentó úlceras genitales, lo que podría explicar la menor frecuencia de esta manifestación clínica al aplicar los criterios BDCJ ( $\mathrm{p}=0,049)$ (Tabla 6). 
Tabla 5. D iferencias de presentación clínica según género $(n=44)$

\begin{tabular}{|lccccc|}
\hline Síntoma & \multicolumn{2}{c}{ H ombre } & \multicolumn{2}{c|}{ M ujer } & $\mathbf{p}$ \\
& $\mathbf{n = 2 5}$ & $\mathbf{( \% )}$ & $\mathbf{n = 1 9}$ & $\mathbf{( \% )}$ & \\
\hline Úlceras orales recurrentes & 23 & 92 & 19 & 100 & 0,595 \\
Úlceras genitales & 17 & 77 & 12 & 63 & 0,498 \\
Compromiso oftalmológico & 16 & 64 & 12 & 63 & 0,804 \\
Lesiones cutáneas & 18 & 72 & 11 & 58 & 0,516 \\
Artritis & 5 & 20 & 5 & 26 & 0,914 \\
Compromiso vascular & 6 & 24 & 7 & 37 & 0,547 \\
Compromiso SNC & 6 & 24 & 5 & 26 & 0,841 \\
Compromiso SNP & 1 & 4 & 2 & 11 & 0,761 \\
\hline
\end{tabular}

$\mathrm{n}$ =número de pacientes, $\mathrm{SNC}=$ sistema nervioso central, $\mathrm{SNP}=$ sistema nervioso periférico.

Tabla 6. M anifestaciones clínicas en 37 pacientes chilenos con enfermedad de Behçet. Clasificación de acuerdo a los criterios diagnósticos BD CJ e ISG

\begin{tabular}{|lrrrrc|}
\hline Síntomas & \multicolumn{2}{c}{ Criterios BD C J } & \multicolumn{2}{c|}{ Criterios ISG } & p \\
& $\begin{array}{l}\text { (completo e incompleto) } \\
\mathbf{n = 3 7}\end{array}$ & $\mathbf{( \% )}$ & $\mathbf{n = 3 2}$ & $\mathbf{( \% )}$ & \\
\hline Úlceras orales & 36 & 97 & 32 & 100 & 0,977 \\
Úlceras genitales & 29 & 78 & 31 & 97 & $0,049^{*}$ \\
Lesiones cutáneas & 27 & 73 & 25 & 78 & 0,841 \\
Compromiso oftalmológico & 27 & 73 & 23 & 72 & 0,859 \\
Manifestaciones articulares & 8 & 22 & 8 & 25 & 0,993 \\
Compromiso vascular & 13 & 35 & 10 & 31 & 0,924 \\
Compromiso SNC & 10 & 27 & 8 & 25 & 0,931 \\
Compromiso SNP & 2 & 5 & 2 & 6 & 0,609 \\
\hline
\end{tabular}

BDCJ =Behçet's Disease Research Committee of Japan. ISG =International Study Group for Behçet's Disease. $\mathrm{n}=$ número de pacientes. * $\mathrm{p}$ significativo $(\mathrm{p}<0,05)$. $\mathrm{SNC}=$ sistema nervioso central. $\mathrm{SNP}=$ sistema nervioso periférico.

El test de patergia fue realizado sólo en 6 pacientes, resultando negativo en 5 de ellos.

Al realizar la comparación de las formas de presentación de nuestros pacientes con una cohorte española y otra griega, encontramos que nuestros pacientes tuvieron una mayor frecuencia de compromiso oftalmológico que los españoles, un mayor compromiso del sistema vascular y del sistema nervioso periférico que los griegos y una menor frecuencia de compromiso articular que ambos grupos (Tabla 7).

\section{DisCUSIÓN}

La EB es poco conocida en general y se manifiesta por una variedad de síntomas y signos, comprometiendo distintos órganos. Según el territorio 


\section{Tabla 7. C aracterísticas clínicas en 3 series de pacientes adultos con enfermedad de Behçet y comparación de cohorte chilena con 2 internacionales}

\begin{tabular}{|c|c|c|c|c|c|}
\hline Síntomas & $\begin{array}{c}\text { España }^{\dagger} \\
(\mathbf{n}=38) \\
\%\end{array}$ & $\begin{array}{c}\text { G recia }^{\ddagger} \\
(\mathbf{n}=82) \\
\%\end{array}$ & $\begin{array}{c}\text { Chile } \\
(n=44) \\
\%\end{array}$ & $\begin{array}{c}\text { España v/s } \\
\text { Chile } \\
\text { p }\end{array}$ & $\begin{array}{c}\text { Grecia v/s } \\
\text { Chile } \\
\text { p }\end{array}$ \\
\hline Úlceras orales & 100 & 100 & 95 & 0,476 & 0,184 \\
\hline Úlceras genitales & 91 & 83 & 75 & 0,108 & 0,401 \\
\hline Lesiones cutáneas & 73 & 73 & 66 & 0,656 & 0,537 \\
\hline Compromiso oftalmológico & 35 & 77 & 64 & $0,016^{*}$ & 0,178 \\
\hline Manifestaciones articulares & 62 & 60 & 23 & $0,000^{*}$ & $0,000^{*}$ \\
\hline Compromiso vascular & 19 & 11 & 30 & 0,372 & $0,015^{*}$ \\
\hline Compromiso SNC & ${ }^{{ }_{N}} \mathrm{NR}$ & 20 & 25 & & 0,583 \\
\hline Compromiso SNP & ${ }^{*}{ }_{N R}$ & 0 & 10,5 & . & $0,01^{*}$ \\
\hline
\end{tabular}

${ }^{\dagger}$ Zouboulis et $\mathrm{al}^{11}$. ${ }^{\ddagger}$ Zouboulis et $\mathrm{al}^{12} .{ }^{{ }} \mathrm{NR}=$ no reportado. ${ }^{*} \mathrm{p}$ significativo $(\mathrm{p}<0,05) . \mathrm{SNC}=$ sistema nervioso central. SNP $=$ sistema nervioso periférico.

afectado puede provocar graves secuelas, especialmente a nivel ocular y de SNC, determinando incapacidad importante o incluso muerte.

A pesar del prolongado período revisado y que los 2 centros involucrados corresponden a instituciones de derivación de patologías de alta complejidad como es la EB, sólo se encontraron 44 casos en 22 años. Si bien esta muestra no permite obtener conclusiones definitivas en relación a la prevalencia de EB en Chile ni de sus manifestaciones clínicas, nuestro estudio nos da una aproximación general de ambas, confirmando la creencia de que la EB tiene baja frecuencia de presentación en nuestro medio. También es probable que exista subdiagnóstico de la enfermedad debido a la variedad de su sintomatología y porque en muchos casos puede presentarse sólo con manifestaciones menores de forma no simultánea, determinando así, la falta de derivación a un especialista.

Al revisar la literatura internacional nos encontramos con que la edad de presentación va desde la infancia hasta más de 78 años, aunque la mayor frecuencia la encontramos entre los 20 y 35 años ${ }^{10}$, lo que es coincidente con nuestra serie en que la edad promedio fue de 26,29 $\pm 11,76$ años. En relación a la distribución por género, en nuestra cohorte no hubo diferencias estadística- mente significativas, lo que es concordante con el cambio epidemiológico que se viene describiendo en los últimos años.

En cuanto a la frecuencia de aparición de los síntomas, se describen las úlceras orales como el más frecuente oscilando en las distintas series entre 96\% y 100\%. En nuestros pacientes la frecuencia fue de $95,5 \%$. Las úlceras genitales y las lesiones cutáneas, también se encontraron en rangos similares a los descritos en otras series ${ }^{11,12}$.

En relación al compromiso oftalmológico destaca, la presencia de panuveítis como la presentación más frecuente al igual que la literatura internacional ${ }^{8}$, lo que es de gran relevancia ya que el número, la severidad y el tratamiento de estos eventos determinarán la extensión del daño estructural que resultará de la pérdida visual irreversible.

Si bien la ocurrencia del compromiso articular fue menor que en otras series internacionales, cuando se presentó, fue severo y de difícil tratamiento.

Ha sido publicado que el neuro-Behçet constituye una manifestación clínica severa con graves secuelas y que disminuye la sobrevida en relación a los que no la presentan ${ }^{13}$. En nuestra serie el compromiso del SNC tuvo similar frecuencia que en series internacionales. Sin embargo, evidencia- 
mos que aquellos que fueron tratados en forma precoz presentaron secuelas menos severas que los pacientes que recibieron tratamiento tardío, los que quedaron con secuelas graves, imposibilitando su reinserción laboral o reincorporación a estudios superiores.

Por otra parte, ha sido reportado que la ciclosporina A podría inducir manifestaciones neurológicas en pacientes con $\mathrm{EB}^{14}$. Once pacientes de nuestra serie presentaron compromiso del SNC, 7 de ellos neurovasculo-Behçet y 4 neuroBehçet. En todos ellos el compromiso neurológico se presentó al inicio del cuadro clínico y precediendo al tratamiento inmunosupresor, por lo que no parece haber una relación de causalidad entre el tratamiento con ciclosporina A y la aparición de los síntomas neurológicos.

Es destacable que en 3 de nuestros casos hubo compromiso de SNP, que si bien está descrito en la literatura, es de muy baja ocurrencia.

$\mathrm{Si}$ bien, algunas de las diferencias en las frecuencias encontradas para cada manifestación clínica con respecto a la literatura internacional pueden explicarse por diferencias reales (etnia, ambiente, etc.), no se puede descartar un sesgo de la muestra, considerando que en los centros estudiados existe importante derivación de patología inflamatoria ocular.

Los criterios BDCJ nos permitieron diagnosticar un mayor número de pacientes que los ISG. Aunque los criterios BDCJ son más exigentes que

\section{REFERENCIAS}

1. Cheng TO. Behçet Disease, Adamantiades-Behçet Disease, or Hippocrates-Adamantiades-Behçet Disease? Chest 2002; 122: 381-2.

2. Susuki M, Susuki N. Behçet's disease. Clin Exp Med 2004; 3: 10-20.

3. Cohen R, Metzger S, Nahir M, Chajek-Shaul T. Association of the MIC-A gene and HLA-B51 with Behçet's disease in Arabs and non-Ashkenazi Jews in Israel. Ann Rheum Dis 2002; 61: 157-60.

4. Cuchacovich M, Merino G, Yamamoto JH, Villarroel F, SAavedra T, Jofré S ET AL. Behçet's disease patients present high levels of deglycosylated anti lipotei- los ISG para el diagnóstico de EB completa (pues requiere de 4 síntomas típicos), ambos criterios son similares para el diagnóstico de EB incompleta. Sin embargo, los criterios ISG dan una mayor importancia a la presencia de úlceras orales (ya que en su ausencia no se puede diagnosticar la enfermedad) mientras que los BDCJ otorgan más relevancia al compromiso ocular. Esta diferencia se puede explicar por el hecho que la EB tiene variabilidad en su expresión clínica en las distintas etnias. Por ejemplo la EB es una de las principales causas de amaurosis en Japón y Turquía, lo que no ocurre en Europa. Otra diferencia es que el test de patergia está incluido en los criterios ISG y no en los BDCJ. En este punto debemos considerar que la aplicación clínica del test de patergia no fue frecuente en nuestro medio y cuando fue realizado en general resultó negativo. Por lo anteriormente expuesto los criterios BDCJ podrían contribuir a disminuir el subdiagnóstico de $\mathrm{EB}$ en nuestro medio.

En síntesis, la EB se caracteriza por la severidad de su expresión clínica en nuestro medio, lo que es concordante con las cohortes internacionales. Por lo anterior parece importante sensibilizar a los distintos profesionales del área de la salud en cuanto a las formas de presentación de la EB, de manera que exista un alto índice de sospecha que permita el diagnóstico precoz, un tratamiento oportuno y evitar así la severa morbimortalidad asociada a esta patología. 
8. Tugal-Tutkun I, Onal S, Altan-Yaycioglu R, Huseyin Altunbas H, Urgancioglu M. Uveitis in Behçet disease: An Analysis of 880 patients. Am J Ophtalmol 2004; 138: 373-80.

9. Merino G, Varas G, Díaz G, Gutiérrez M, Massardo L, Pacheco D ET al. Eficacia del infliximab en pacientes con síndrome de Behçet portadores de úveo-retinitis grave. Rev Méd Chile 2006; 134: 875-82.

10. Kaklamani V, Vaiopoulos G, Kaklamanis P. Behçet's disease. Sem Arth Rheum 1998; 27: 197-217.

11. Zouboulis C, Kotter I, Djawari D, Kirch W, Kohl P, Ochsendorf F ET AL. Epidemiological Features of Adamantiades-Behçet's Disease in Germany and in Europe. Yonsei Med J 1997; 38: 411-22.
12. Zouboulis C, Vaiopoulos G, Marcomichelakis N, Palimeris G, Markidou I, Thouas B et al. Onset signs, clinical course, prognosis, treatment and outcome of adult patients with Adamantiades-Behçet's disease in Greece. Clin Exp Rheum 2003; 21(suppl 30): S19-26.

13. Akman-Demir G, Serdaroglu P, Tasçi B, and the Neuro BehÇET Study Group. Clinical patterns of neurological involvement in Behçet's disease: evaluation of 200 patients. Brain 1999; 122: 2171-81.

14. Kotake S, Higashi K, Yoshikawa K, Sasamoto Y, Окамото T, Matsuda H. Central nervous system symptoms in patients with Behçet disease receiving cyclosporine therapy. Ophthalmology 1999; 106: 586-9. 